\title{
Electron Transmission through Coordinating Atoms Embedded in Metal-Organic Nanoporous Networks
}

\author{
Ignacio Piquero-Zulaica ${ }^{1,2,3,{ }^{*}}$ Ali Sadeghi $\odot,{ }^{4,5, \dagger}$ Mohammad Kherelden, ${ }^{6}$ Muqing Hua $\odot,{ }^{7}$ Jing Liu $\odot,{ }^{7}$ \\ Guowen Kuang, ${ }^{7}$ Linghao Yan $\odot,{ }^{7}$ J. Enrique Ortega, ${ }^{1,2,8}$ Zakaria M. Abd El-Fattah $\odot,{ }^{6}$ \\ Behnam Azizi, ${ }^{4}$ Nian Lin, ${ }^{7}$ and Jorge Lobo-Checa $\odot^{9,10, \$}$ \\ ${ }^{1}$ Centro de Física de Materiales CSIC/UPV-EHU-Materials Physics Center, Manuel Lardizabal 5, E-20018 San Sebastián, Spain \\ ${ }^{2}$ Donostia International Physics Center, Paseo Manuel Lardizabal 4, E-20018 Donostia-San Sebastián, Spain \\ ${ }^{3}$ Physik Department E20, Technische Universität München, 85748 Garching, Germany \\ ${ }^{4}$ Department of Physics, Shahid Beheshti University, GC, Evin, 19839 Tehran, Iran \\ ${ }^{5}$ School of Nano Science, Institute for Research in Fundamental Sciences (IPM), 19395-5531 Tehran, Iran \\ ${ }^{6}$ Physics Department, Faculty of Science, Al-Azhar University, Nasr City, E-11884 Cairo, Egypt \\ ${ }^{7}$ Department of Physics, The Hong Kong University of Science and Technology, Hong Kong, China \\ ${ }^{8}$ Universidad del País Vasco, Dpto. Física Aplicada I, E-20018 San Sebastián, Spain \\ ${ }^{9}$ Instituto de Ciencia de Materiales de Aragón (ICMA), CSIC-Universidad de Zaragoza, E-50009 Zaragoza, Spain \\ ${ }^{10}$ Departamento de Física de la Materia Condensada, Universidad de Zaragoza, E-50009 Zaragoza, Spain
}

(Received 29 July 2019; published 31 December 2019)

\begin{abstract}
On-surface metal-organic nanoporous networks generally refer to adatom coordinated molecular arrays, which are characterized by the presence of well-defined and regular nanopores. These periodic structures constructed using two types of components confine the surface electrons of the substrate within their nanocavities. However, the confining (or scattering) strength that individual building units exhibit is a priori unknown. Here, we study the modification of the substrate's surface electrons by the interaction with a $\mathrm{Cu}$-coordinated TPyB metal-organic network formed on $\mathrm{Cu}(111)$ and disentangle the scattering potentials and confinement properties. By means of STM and angle-resolved photoemission spectroscopy we find almost unperturbed free-electron-like states stemming from the rather weak electron confinement that yields significant coupling between adjacent pores. Electron plane wave expansion simulations match the superlattice induced experimental electronic structure, which features replicating bands and energy renormalization effects. Notably, the electrostatic potential landscape obtained from our ab initio calculations suggests that the molecules are the dominant scattering entities while the coordination metal atoms sandwiched between them act as leaky channels. These metal atom transmission conduits facilitate and enhance the coupling among quantum dots, which are prone to be exploited to engineer the electronic structure of surface electron gases.
\end{abstract}

DOI: $10.1103 /$ PhysRevLett.123.266805

Nanoporous networks grown on noble metals have been established in the last decade as reliable confining entities for the surface state (SS) electrons [1-4]. Each pore acts as a quantum dot (QD) and their properties are tunable by proper selection of the building units. These building units dictate the effective potential barriers [5-7], the geometry of the array [4], and the existing interaction with the substrate $[7,8]$ defining the ultimate confining capabilities of the system. Metal-organic nanoporous networks (MONNs) are possibly the most versatile and robust of noncovalent organic arrays when compared to other related electrostatically bonded (hydrogen or halogen) networks [1-4,9-11]. Their stability can be utilized for condensing guest organic and inorganic adsorbates [12-17] and their metal coordination atoms show novel magnetic properties $[18,19]$, catalytic effects [20], and variable valence oxidation states [21]. Moreover, different tessellation patterns can be tailored (hexagonal, square, or quasicrystalline) when rationally playing with the molecular end groups (cyano, pyridyl), coordination atoms (transition metal, rare earth) or the support surface (noble metal, $h \mathrm{BN}$, graphene) [22-24], even resulting in theoretically predicted exotic topological properties [25-28].

The atomically precise regularity of MONNs allows the investigation of electron confinement and periodic QD intercoupling that strongly depends on the building units' potential landscape. These quantum effects manifest as energy shifts and gap openings at high symmetry points in the electronic bands when studied by angle-resolved photoemission spectroscopy (ARPES) $[2,4,29,30]$. Such modifications to the two-dimensional electron gas (2DEG) have been reported for several organic and metal-organic nanoporous networks and show flexibility upon organic unit selection $[1,2,7,31]$ by affecting the pore dimensions 
(QD size) [3,6] and/or the barrier widths [4]. The resulting QD array bands respond to thermal effects [32] and to the condensation of weakly interacting guest atoms and molecules within their pores [33].

Unlike the well-understood scattering mechanisms of quantum corrals [34] and step edges [35,36], the scattering properties of molecules and metal atoms generating MONNs are not yet clear. The evidence so far suggests smaller potential barriers of the coordinating single metal atoms than the molecules [3,6,37]. This confinement strength exerted upon the 2DEG is found to vary from one network to another, necessarily affecting the electronic intercoupling between neighboring QDs.

In this Letter, we provide insight into the potential landscape of a MONN by disentangling the scattering properties of the molecules and single coordination atoms. We confirm that metal adatoms act as weak scatterers compared to the organic molecular backbones and, due to their strategic position within the array, they provide accessible transmission channels for the surface electrons, further enhancing the interpore coupling. Such findings rely upon mesoscopic scale (by means of the spatially averaging ARPES) and nanoscale [by highly resolved scanning tunneling microscopy (STM) and spectroscopy (STS)] experimental electronic characterization of the MONN supported theoretically by electron plane wave expansion (EPWE) simulations and electrostatic potential (ESP) maps calculations using density functional theory (DFT).

The studied semiconducting $\mathrm{Cu}$-coordinated network, that we name hereafter TPyB-Cu, is shown in Fig. 1(a). It is generated from thermally evaporated TPyB $[1,3,5$-tri(4pyridyl)-benzene], sketched in the figure inset. The precursors are deposited at $\mathrm{RT}$ on $\mathrm{Cu}(111)$ and annealed to $420 \mathrm{~K}$, leading to a periodic, long-range ordered and singledomain (of the substrate's average terrace size), and practically defect-free network. This QD array is thermally robust up to $600 \mathrm{~K}$ [38]. Such a homogeneous formation only happens when the MONN saturates the surface (two differently oriented phases coexist for submonolayer coverages [8]). As shown in Fig. 1(b), the $\mathrm{Cu}$ coordination atoms bond to the pyridyl groups of TPyB in a twofold coordination that presents sixfold symmetry [8]. Constant current STM imaging shows that the apparent height at adatom positions is significantly smaller than at the molecular centers, suggesting that they are closer to the surface than the average molecular backbones. The pore-to-pore periodicity is $2.65 \mathrm{~nm}$ along the [112]] direction resulting in a $(6 \sqrt{3} \times 6 \sqrt{3}) R 30^{\circ}$ superstructure [cf. LEED pattern in Fig. 1(c)]. The nanopore areas of $6 \mathrm{~nm}^{2}$ should confine surface electrons [7]; therefore, we probe them by ARPES and STS.

Figures 2(a)-2(c) show the experimental ARPES spectral density of the pristine $\mathrm{Cu}$ SS and the TPyB-Cu covered surface along the two network high-symmetry directions (rotated $30^{\circ}$ from the substrate's main directions). We

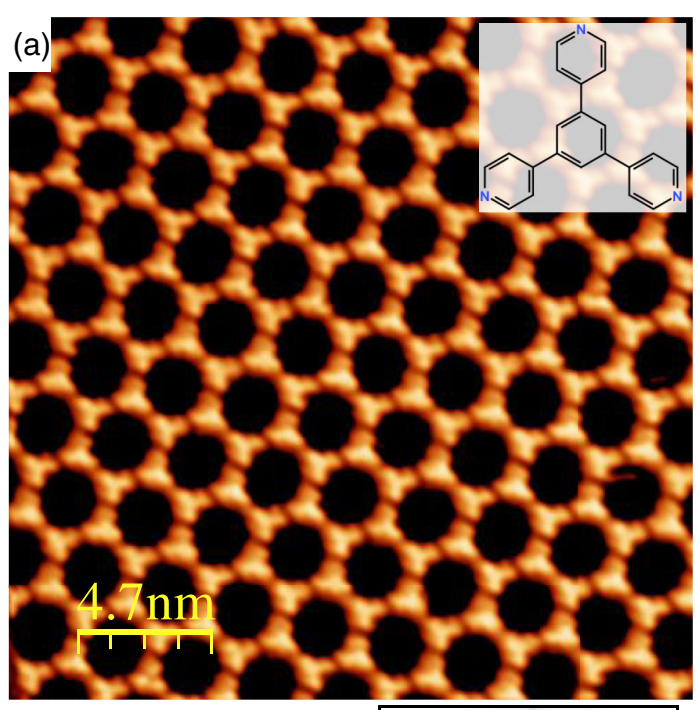

(b)

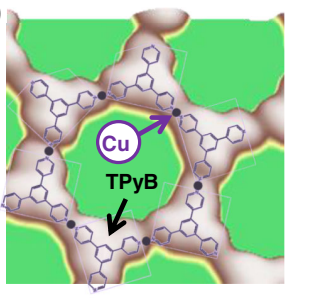

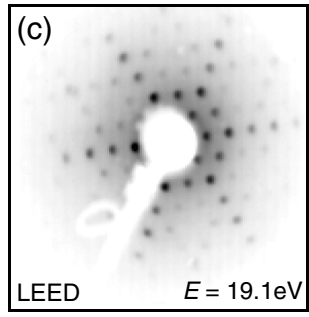

FIG. 1. Topographic characterization of the TPyB-Cu metalorganic network. (a) High-resolution STM image of the singledomain $\mathrm{Cu}$-coordinated TPyB-Cu hexagonal QD array grown on $\mathrm{Cu}(111)$. The inset shows the precursor molecule. (b) Enlargement into the hexagonal porous structure with a superimposed structural model. (c) LEED pattern of the network, which shows evidence that the molecular film is long-range ordered and single domain on the $\mathrm{Cu}(111)$ surface.

observe a down-shift of the fundamental energy ( $\bar{\Gamma}$ point $)$ of $-70 \mathrm{meV}$, that becomes obvious after comparing the normal emission energy distribution curves (EDCs) of Fig. 2(d) (cf. Table SI of the Supplemental Material [39]). Such a counterintuitive fundamental energy shift is explained elsewhere [6] and is also found for other related MONNs generated with larger precursors (see Fig. S1). Note that in this ARPES energy window only the modified 2DEG state is found, validating the semiconducting character of the metal-organic overlayer (see Figs. S2-S4) [8].

This band structure should correlate to the local density of states (LDOS) when probed by STS. Figure 2(e) shows position-dependent experimental conductance $(d I / d V)$ spectra for TPyB-Cu that are compared to the $\mathrm{Cu}$ SS. As expected from ARPES, the $d I / d V$ onsets of the TPyB$\mathrm{Cu}$ are also shifted by $-70 \mathrm{meV}$ with respect to the $\mathrm{Cu}$ SS (dashed vertical lines). Moreover, the $d I / d V$ line shape at the pore center (red line) shows weak confinement resonances peaking at $\sim-0.42 \mathrm{~V}(n=1$ state $), \sim-0.23 \mathrm{~V}$ ( $n=2,3$ states) and $\sim 0.2 \mathrm{~V}(n=4$ state), characteristic of trapped electrons [1-4,17,33]. This identification of the LDOS features allows us to explicitly correct the 

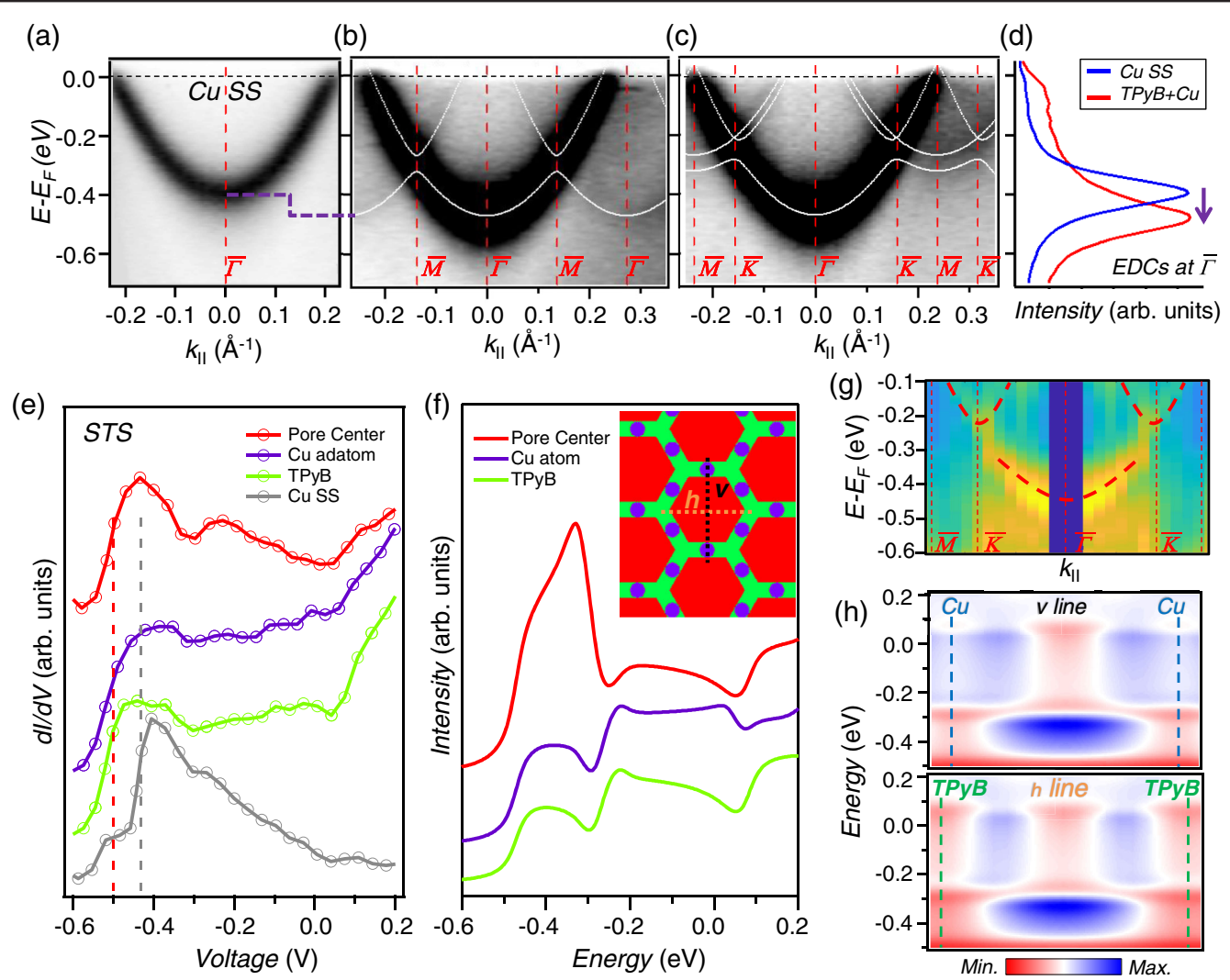

FIG. 2. Electronic structure of the TPyB-Cu nanoporous network. Top row contains ARPES experimental datasets obtained at $150 \mathrm{~K}$ that compares the 2DEG of the pristine surface (a) with the TPyB-Cu network along the two high-symmetry directions: (b) $\overline{\Gamma M}$ and (c) $\overline{\Gamma K}$. A down-shift of the band bottom (see dashed purple line) and replicating bands following the superperiodicity of the MONN (vertical dashed red lines) are observed. (d) EDCs at normal emission ( $\bar{\Gamma}$ point) for TPyB-Cu (red) exhibits a down-shift of -70 meV with respect to the pristine $\mathrm{Cu} S \mathrm{SS}$ (blue). (e) Local electronic structure at different MONN positions shown as STS $d I / d V$ spectra for the pore center (red), the molecule (green) and the $\mathrm{Cu}$ coordination atom (purple). These are simulated in (f) by EPWE as solid lines of the same colours using the scattering geometry depicted in the inset $\left(V_{\text {mol }}=250 \mathrm{meV}\right.$ and $\left.V_{\mathrm{Cu}}=50 \mathrm{meV}\right)$. The down-shift of the experimental $d I / d V \mathrm{~s}$ [red vs gray vertical dashed lines in (e)] agrees with the ARPES observations in the upper row and is correctly reproduced by our EPWE simulations [overlaid white lines in (b) and (c) indicate EPWE band calculations]. (g) Power spectral functions of $2 \mathrm{DEG}$ along $\mathrm{Cu}$ to $\mathrm{Cu}$ direction that resembles the ARPES band structure along the $\overline{\Gamma K}$ direction shown in (c). (h) EPWE simulated 1D LDOS maps along the horizontal (molecule to molecule) and vertical $(\mathrm{Cu}$ to $\mathrm{Cu}$ ) directions outlined in the inset of (f).

assignment of the $\mathrm{S} 1$ peak in Ref. [7] as the $n=2,3$ states. The 2DEG confinement at the pores is unraveled in ARPES by the presence of small gaps at symmetry points [practically invisible in the raw data of Figs. 2(b) and 2(c)]. However, the replica bands in adjoining Brillouin zones and small energy gaps (see Figs. S2-S4), imply weak 2DEG scattering from the network barriers.

To shed light into the surface potential landscape that TPyB-Cu entails for the 2DEG, we perform EPWE simulations. This semiempirical method has been successfully used to obtain the confining character of related nanoporous networks [1,3-5]. Our simulations agree simultaneously with the ARPES and STS datasets when using the geometry shown in the inset of Fig. 2(f) (constructed from the chemical model structure of the MONN) and assuming two different scattering regions: highly repulsive for molecules $\left(V_{\mathrm{mol}}=250 \mathrm{meV}\right.$ in green) and weakly repulsive around the $\mathrm{Cu}$ adatoms
$\left(V_{\mathrm{Cu}}=50 \mathrm{meV}\right.$ in dark blue) $[1,3,5]$ (Fig. S5 shows the effect in the band structure when tweaking these potential values). Using the modified 2DEG parameters indicated in Table SI for the EPWE simulations, we can nicely reproduce the experimental ARPES replicating bands [white lines in Figs. 2(b) and 2(c)] and also the spectral intensity and weak scattering strength of the networks (see Fig. S4). Moreover, we find good agreement with the experimental conductance spectra in the simulated line shapes, as shown in Fig. 2(f) (the down-slope experimental background is not included in our simulations). The LDOS at the molecule and $\mathrm{Cu}$ adatoms show steplike onsets that are weakly modulated up to $0.2 \mathrm{~V}$ corresponding to the modified 2DEG. Molecular states are observed much further away from the Fermi energy [8]. We note that no bound state feature is found at the $\mathrm{Cu}$ adatom spectrum, contrarily to the case of isolated adatoms on pristine noble metal surfaces $[47,48]$. The experimental agreement 
between ARPES and STS is also manifested by the similarity between the power spectral functions along the pore-to-pore direction [Fig. 2(g)] and the ARPES band structure along $\overline{\Gamma K}$ [Fig. 2(c)]. The discontinuous red lines overlaid as a guide to the eye closely resemble the gray scale electronic structure shown in ARPES.

The EPWE permits us to simulate one-dimensional (1D) linescans along the horizontal and vertical directions, as shown in Fig. 2(h). At the $\mathrm{Cu}$ adatom positions (vertical direction) weaker barriers than at the molecular units are felt by the 2DEG. Even when the first confined state $(n=1)$ is well encapsulated in both directions within the pore, we hypothesize that higher resonances effectively leak through the $\mathrm{Cu}$ barriers ( $v$ line) and smoothly couple adjoining pores. Such weak scattering originates at the metal-organic coordination position, strategically located between nanopores. As a result, the free-electron-like character of the pristine 2DEG becomes almost unperturbed in comparison to other studied organic or metalorganic networks $[2,4]$, where appreciable energy gaps were discernible.

To verify the hypothesis that single metal atoms present weaker scattering potentials for the surface electrons than the molecular backbones, we extract the electrostatic potential (ESP) maps from DFT calculations for the TPyB-Cu network. First, we generate the network and calculate the scattering potential landscape encountered by surface electrons at selected planes $(z)$ from the average MONN position, which is taken as a reference (see Fig. S6). Since the $\mathrm{Cu}(111)$ SS has its largest probability density close to the substrate's last layer atomic centers and exponentially decays towards the vacuum [49], we plot the resulting ESP maps at the atomic centers' plane $(z=3.08 \AA)$ and halfway in their atomic radius $(z=2.36 \AA)$ in Figs. 3(a) and 3(b) (see 3D perspective in Fig. S7). Notably, our calculations exhibit remarkable agreement with the scattering potential landscape used in EPWE simulations and corroborates the heterogeneous scattering potential that features strong repulsive character at the molecules [4,50-52] and quite weak at $\mathrm{Cu}$ coordination positions. Inspection into the potential line profiles extracted from these calculations, shows that the $\mathrm{Cu}$ adatom regions present a particular barrier substructure. At these sites, the outer rim is weakly repulsive, but quickly reverses its character towards its center. We find that such behavior is $z$ dependent, becoming more pronounced as we move away from the atomic centers' plane. This observation validates our hypothesis that the $\mathrm{Cu}$ adatoms act like effective transmission channels between adjacent nanopores, preventing the appearance of noticeable energy gaps and strong confinement features in our ARPES and STS datasets.

For comparison, we calculate the ESP maps for the reported 3,9-dibromodinaphtho[2,3-b:2', 3'-d]thiophene (Br-DNT) halogen bonded nanoporous network grown on $\operatorname{Ag}(111)$ [4]. We select this molecular array since it bears a similar interpore separation and QD size, but
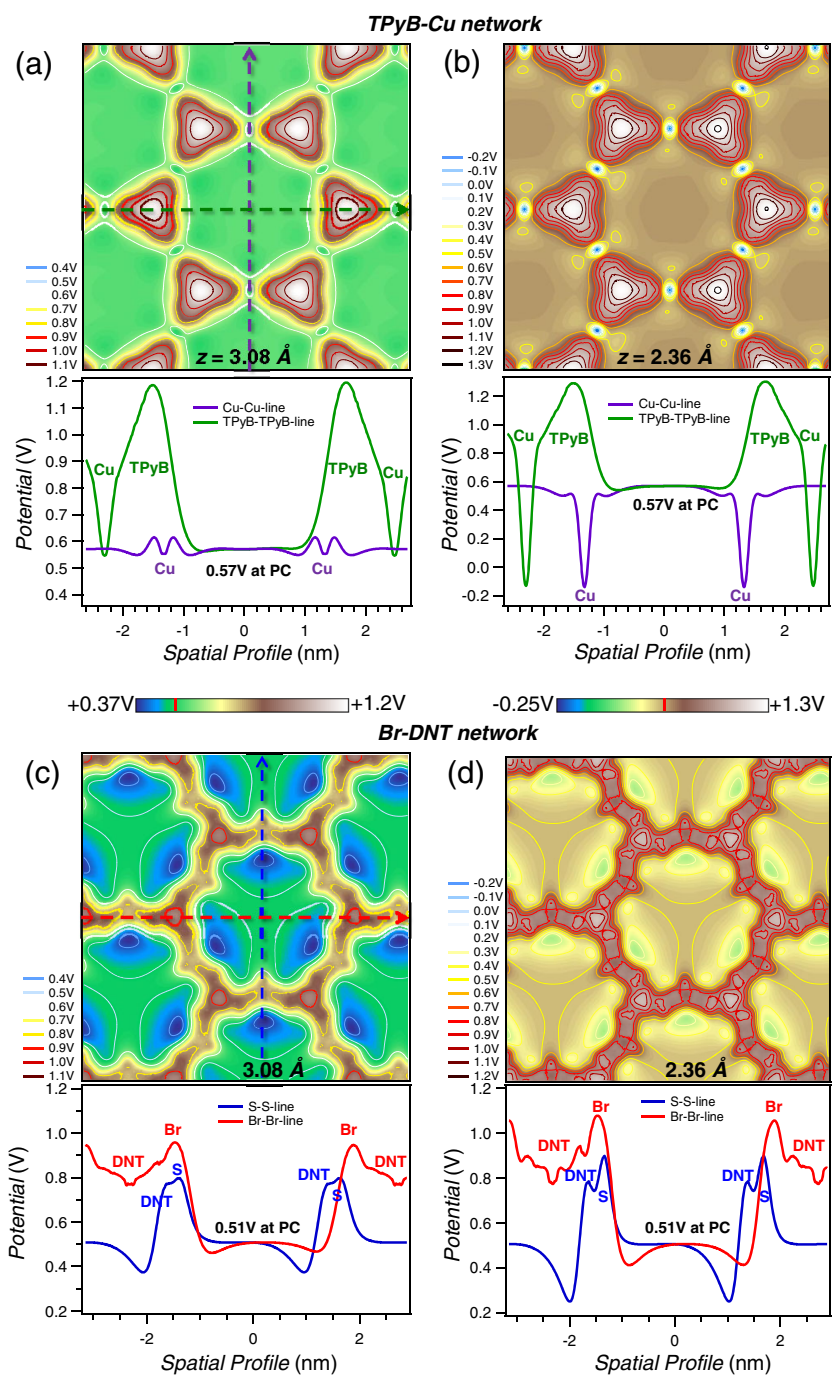

FIG. 3. Electrostatic potential map calculations for TPyB-Cu [panels (a) and (b)] and comparison to the hologen-bonded BrDNT network from Ref. [4] [panels (c) and (d)]. On the top of the panels, electrostatic potential contour plot maps corresponding to $3.08 \AA$ (last surface layer) [(a) and (c)] and $2.36 \AA$ planes [(b) and (d)] with respect to the average molecular backbone are shown. Beneath these, two perpendicular potential line profiles are extracted for each case, revealing the significant potential difference encountered by the 2DEG: $\mathrm{Cu}$ adatoms present conductive channels in TPyB-Cu, which are absent in the better confining Br-DNT network.

lacks the metal coordination. Even if there are slight site dependent differences $(\mathrm{Br}$ and $\mathrm{S}$ atoms show stronger repulsion character than the $\mathrm{C}$ backbone), our calculations displayed in Figs. 3(c) and 3(d) show a very homogeneous repulsive potential barrier landscape compared to the TPyB-Cu case. In essence, the Br-DNT network efficiently confines the electrons within the nanopores at different $z$ planes. Since there is no transmission channel for surface electrons to leak through, the QD intercoupling is relatively hindered and noticeable energy gaps and band flattening is found in this network. This takes place even when the 
reported EPWE potential for the carbon backbone is half the value of the TPyB-Cu network reported here. Note that no down-shift of the fundamental energy is present on Br-DNT [4] (see Table SI), confirming its origin in the coordinating metal adatoms [6]. Importantly, further EPWE simulations show that the side position of the coordinating adatoms is key for opening a transmission channel between neighboring pores, since this effect is absent at corner positions (see Fig. S8).

Nevertheless, we find an important difference in the absorption geometry between the two networks. While the Br-DNT layer stays practically flat at $0.3 \mathrm{~nm}$ distance from the substrate [4], the ТPyB molecules significantly bend when coordinating with the $\mathrm{Cu}$ adatoms [8] and come as close as $0.2 \mathrm{~nm}$ to the substrate surface at their edges (see Fig. S6). Note that such bending is also found for Br-DNT when forming metal-organic coordination chains with $\mathrm{Ag}$ adatoms [53]. In any case, the large atomic radius of the coordinating adatoms enhance their orbital hybridization with the substrate. On this basis, the role of the bridging $\mathrm{Cu}$ adatoms that facilitates the interpore electron transfer and the fundamental energy down-shifts, become physically justified.

In summary, we have grown a single-domain and longrange ordered TPyB-Cu MONN network film that saturates the $\mathrm{Cu}(111)$ surface. The electronic structure modification of the substrate's SS was studied through a combination of experimental ARPES and STS together with EPWE simulations and DFT based ESP map calculations. Based on the practical absence of energy gaps and unmodified effective mass, we find that the coordination metal atoms exhibit the weakest potential barriers for the surface electrons, which practically spoil the confining properties of their molecular counterparts. Since these $\mathrm{Cu}$ adatoms are located at strategic positions of the network, they act like efficient transmission channels between adjacent pores that yields significant interdot coupling. In this way, they provide leakage pathways that severely weaken the pore confining capabilities. In opposition to the repulsive character of molecular barriers, our ESP maps show that metal coordination atoms activate such transmission channels by spatially varying their character from slightly repulsive at the outer rim to attractive at their cores. Contrarily to these single adatom coordination nanoporous networks, homomolecular arrays (like Br-DNT) present more homogeneous potential barriers that enhance confinement effects and reduce the interdot coupling.

Our insight into the potential landscape of such 2D nanostructures could trigger further interest in tailoring the confinement properties of these networks. Particularly, it could contribute to extend its use to other molecules and coordinating elements, such as rare earths and alkali metals, grown on different types of surfaces, such as topological insulators or Rashba type surface alloys that host topologically protected or spin textured helical surface states.
We thank J. García de Abajo for providing the EPWE code and the computational resources provided by the SARMAD cluster of SBU. Financial support is acknowledged from the Spanish Ministry of Economy, Industry and Competitiveness (MINECO, Grants No. MAT2016-78293C6 and No. FIS2016-75862-P), from the regional Government of Aragon (Grant No. E12-17R), from the Basque Government (Grant No. IT-1255-19), from the European Regional Development Fund (ERDF) under the program Interreg V-A España-Francia-Andorra (Contract No. EFA 194/16 TNSI) and from the Hong Kong RGC 16304016.

* Corresponding author. ipiquerozulaica@gmail.com † Corresponding author. ali_sadeghi@sbu.ac.ir Corresponding author. jorge.lobo@csic.es

[1] F. Klappenberger, D. Kühne, W. Krenner, I. Silanes, A. Arnau, F. J. García de Abajo, S. Klyatskaya, M. Ruben, and J. V. Barth, Nano Lett. 9, 3509 (2009).

[2] J. Lobo-Checa, M. Matena, K. Müller, J. H. Dil, F. Meier, L. H. Gade, T. A. Jung, and M. Stöhr, Science 325, 300 (2009).

[3] F. Klappenberger, D. Kühne, W. Krenner, I. Silanes, A. Arnau, F. J. García de Abajo, S. Klyatskaya, M. Ruben, and J. V. Barth, Phys. Rev. Lett. 106, 026802 (2011).

[4] I. Piquero-Zulaica, J. Lobo-Checa, A. Sadeghi, Z. M. Abd El-Fattah, C. Mitsui, T. Okamoto, R. Pawlak, T. Meier, A. Arnau, J. E. Ortega et al., Nat. Commun. 8, 787 (2017).

[5] I. Piquero-Zulaica, Z. M. Abd El-Fattah, O. Popova, S. Kawai, S. Nowakowska, M. Matena, M. Enache, M. Stöhr, A. Tejeda, A. Taleb et al., New J. Phys. 21, 053004 (2019).

[6] I. Piquero-Zulaica, J. Li, Z. M. Abd El-Fattah, L. Solianyk, I. Gallardo, L. Monjas, A. K. H. Hirsch, A. Arnau, J. E. Ortega, M. Stöhr et al., Nanoscale 11, 23132 (2019).

[7] S. Wang, W. Wang, L. Z. Tan, X. G. Li, Z. Shi, G. Kuang, P. N. Liu, S. G. Louie, and N. Lin, Phys. Rev. B 88, 245430 (2013).

[8] W. Wang, X. Shi, S. Wang, J. Liu, M. A. Van Hove, P. N. Liu, R.-Q. Zhang, and N. Lin, Phys. Rev. Lett. 110, 046802 (2013).

[9] G. Pawin, K. L. Wong, K.-Y. Kwon, and L. Bartels, Science 313, 961 (2006).

[10] L. Dong, Z. Gao, and N. Lin, Prog. Surf. Sci. 91, 101 (2016).

[11] U. Schlickum, R. Decker, F. Klappenberger, G. Zoppellaro, S. Klyatskaya, M. Ruben, I. Silanes, A. Arnau, K. Kern, H. Brune, and J. V. Barth, Nano Lett. 7, 3813 (2007),

[12] M. Stöhr, M. Wahl, H. Spillmann, L. Gade, and T. Jung, Small 3, 1336 (2007).

[13] Z. Cheng, J. Wyrick, M. Luo, D. Sun, D. Kim, Y. Zhu, W. Lu, K. Kim, T. L. Einstein, and L. Bartels, Phys. Rev. Lett. 105, 066104 (2010).

[14] J. Wyrick, D.-H. Kim, D. Sun, Z. Cheng, W. Lu, Y. Zhu, K. Berland, Y. S. Kim, E. Rotenberg, M. Luo et al., Nano Lett. 11, 2944 (2011). 
[15] S. Nowakowska, A. Wäckerlin, S. Kawai, T. Ivas, J. Nowakowski, S. Fatayer, C. Wäckerlin, T. Nijs, E. Meyer, J. Björk et al., Nat. Commun. 6, 6071 (2015).

[16] R. Zhang, G. Lyu, C. Chen, T. Lin, J. Liu, P. N. Liu, and N. Lin, ACS Nano 9, 8547 (2015).

[17] M. Pivetta, G. E. Pacchioni, U. Schlickum, J. V. Barth, and H. Brune, Phys. Rev. Lett. 110, 086102 (2013).

[18] N. Abdurakhmanova, T.-C. Tseng, A. Langner, C. S. Kley, V. Sessi, S. Stepanow, and K. Kern, Phys. Rev. Lett. 110, 027202 (2013).

[19] T. R. Umbach, M. Bernien, C. F. Hermanns, A. Krüger, V. Sessi, I. Fernandez-Torrente, P. Stoll, J. I. Pascual, K. J. Franke, and W. Kuch, Phys. Rev. Lett. 109, 267207 (2012).

[20] R. Gutzler, S. Stepanow, D. Grumelli, M. Lingenfelder, and K. Kern, Acc. Chem. Res. 48, 2132 (2015).

[21] Y. Li, J. Xiao, T. E. Shubina, M. Chen, Z. Shi, M. Schmid, H.-P. Steinrück, J. M. Gottfried, and N. Lin, J. Am. Chem. Soc. 134, 6401 (2012).

[22] J. I. Urgel, D. Écija, G. Lyu, R. Zhang, C.-A. Palma, W. Auwärter, N. Lin, and J. V. Barth, Nat. Chem. 8, 657 (2016).

[23] L. Yan, G. Kuang, Q. Zhang, X. Shang, P. N. Liu, and N. Lin, Faraday Discuss. 204, 111 (2017).

[24] Y.-Q. Zhang, M. Paszkiewicz, P. Du, L. Zhang, T. Lin, Z. Chen, S. Klyatskaya, M. Ruben, A. P. Seitsonen, J. V. Barth et al., Nat. Chem. 10, 296 (2018).

[25] X. Zhang and M. Zhao, Sci. Rep. 5, 14098 (2015).

[26] L. Z. Zhang, Z. F. Wang, B. Huang, B. Cui, Z. Wang, S. X. Du, H.-J. Gao, and F. Liu, Nano Lett. 16, 2072 (2016).

[27] A. Kumar, K. Banerjee, A. S. Foster, and P. Liljeroth, Nano Lett. 18, 5596 (2018).

[28] Z. Gao, C.-H. Hsu, J. Liu, F.-C. Chuang, R. Zhang, B. Xia, H. Xu, L. Huang, Q. Jin, P. N. Liu et al., Nanoscale 11, 878 (2019).

[29] A. Mugarza, F. Schiller, J. Kuntze, J. Cordón, M. Ruiz-Osés, and J. E. Ortega, J. Phys. Condens. Matter 18, S27 (2006).

[30] A. Bendounan, F. Forster, J. Ziroff, F. Schmitt, and F. Reinert, Surf. Sci. 600, 3865 (2006).

[31] A. Shchyrba, S. C. Martens, C. Wäckerlin, M. Matena, T. Ivas, H. Wadepohl, M. Stöhr, T. A. Jung, and L. H. Gade, Chem. Commun. (Cambridge) 50, 7628 (2014).

[32] I. Piquero-Zulaica, S. Nowakowska, J. E. Ortega, M. Stöhr, L. H. Gade, T. A. Jung, and J. Lobo-Checa, Appl. Surf. Sci. 391, 39 (2017).

[33] S. Nowakowska, A. Wäckerlin, I. Piquero-Zulaica, J. Nowakowski, S. Kawai, C. Wäckerlin, M. Matena, T. Nijs, S. Fatayer, O. Popova et al., Small 12, 3741 (2016).

[34] M. F. Crommie, C. P. Lutz, and D. M. Eigler, Science 262, 218 (1993).
[35] L. Bürgi, O. Jeandupeux, A. Hirstein, H. Brune, and K. Kern, Phys. Rev. Lett. 81, 5370 (1998).

[36] J. E. Ortega, J. Lobo-Checa, G. Peschel, S. Schirone, Z. M. Abd El-Fattah, M. Matena, F. Schiller, P. Borghetti, P. Gambardella, and A. Mugarza, Phys. Rev. B 87, 115425 (2013).

[37] N. Kepčija, T.-J. Huang, F. Klappenberger, and J. V. Barth, J. Chem. Phys. 142, 101931 (2015).

[38] J. Liu, T. Lin, Z. Shi, F. Xia, L. Dong, P. N. Liu, and N. Lin, J. Am. Chem. Soc. 133, 18760 (2011).

[39] See Supplemental Material at http://link.aps.org/ supplemental/10.1103/PhysRevLett.123.266805 for the supplemental methods, table, and figures, which includes Refs. [40-46].

[40] F. J. García de Abajo, J. Cordón, M. Corso, F. Schiller, and J. E. Ortega, Nanoscale 2, 717 (2010).

[41] Z. M. Abd El-Fattah, M. A. Kher-Elden, I. Piquero-Zulaica, F. J. Garcia de Abajo, and J. E. Ortega, Phys. Rev. B 99, 115443 (2019).

[42] L. Genovese, A. Neelov, S. Goedecker, T. Deutsch, S. A. Ghasemi, A. Willand, D. Caliste, O. Zilberberg, M. Rayson, A. Bergman et al., J. Chem. Phys. 129, 014109 (2008).

[43] L. Genovese, T. Deutsch, A. Neelov, S. Goedecker, and G. Beylkin, J. Chem. Phys. 125, 074105 (2006).

[44] C. Hartwigsen, S. Gœdecker, and J. Hutter, Phys. Rev. B 58, 3641 (1998).

[45] Q. Hill and C.-K. Skylaris, Proc. R. Soc. A 465, 669 (2009).

[46] K. Momma and F. Izumi, J. Appl. Crystallogr. 44, 1272 (2011).

[47] F. E. Olsson, M. Persson, A. G. Borisov, J.-P. Gauyacq, J. Lagoute, and S. Fölsch, Phys. Rev. Lett. 93, 206803 (2004).

[48] L. Limot, E. Pehlke, J. Kröger, and R. Berndt, Phys. Rev. Lett. 94, 036805 (2005).

[49] P. M. Echenique, R. Berndt, E. V. Chulkov, T. Fauster, A. Goldmann, and U. Höfer, Surf. Sci. Rep. 52, 219 (2004).

[50] L. Gross, F. Moresco, L. Savio, A. Gourdon, C. Joachim, and K.-H. Rieder, Phys. Rev. Lett. 93, 056103 (2004).

[51] Y. Pennec, W. Auwärter, A. Schiffrin, A. Weber-Bargioni, A. Riemann, and J. V. Barth, Nat. Nanotechnol. 2, 99 (2007).

[52] K. Seufert, W. Auwärter, F. J. García de Abajo, D. Ecija, S. Vijayaraghavan, S. Joshi, and J. V. Barth, Nano Lett. 13, 6130 (2013).

[53] S. Kawai, A. Sadeghi, T. Okamoto, C. Mitsui, R. Pawlak, T. Meier, J. Takeya, S. Goedecker, and E. Meyer, Small 12, 5303 (2016). 